\title{
Conduta do tratamento medicamentoso por cuidadores de idosos
}

Débora Santos Lula Barros ${ }^{(a)}$ Dayde Lane Mendonça Silva(b)

Silvana Nair Leite ${ }^{(c)}$

Barros DSL, Mendonça-Silva DL, Leite SN. Management of drug therapy by elderly people's caregivers. Interface (Botucatu). 2015; 19(54):527-36.

Aging of the population is increasing the number of caregivers of elderly people in Brazil. Pharmacotherapy involves a complex system of activities and its management is among the tasks performed these caregivers. Moreover, management of drug therapy may be subject to various problems relating to its implementation. In particular, there are difficulties in drug administration, lack of access to drugs, obstacles against conducting clinical observation and challenges in establishing effective communication between elderly people and healthcare professionals. The present study consists of a review that aims to expand the discussions on the abovementioned issues so as to provide theoretical support to programs and services within the field of gerontology.

Keywords: Drugs. Elderly people. Caregivers. Management of drug therapy.
O envelhecimento populacional está ampliando o número de cuidadores de idosos no Brasil. A farmacoterapia compreende um sistema complexo de atividades e a sua condução está presente no rol de tarefas desempenhadas por esses atores. Além disso, a conduta do tratamento pode estar sujeita a diversos problemas na sua execução, com destaque para: dificuldades de administração dos medicamentos, falta de acesso aos medicamentos, obstáculos na realização de observação clínica, assim como os desafios presentes no estabelecimento de uma comunicação efetiva com os idosos e profissionais de saúde. O presente estudo trata de uma revisão cujo objetivo é ampliar as discussões sobre os aspectos supracitados, de modo que este debate dê subsídios teóricos aos programas e serviços na área de gerontologia.

Palavras-chave: Medicamentos. Idoso. Cuidadores. Conduta do tratamento medicamentoso. (a) Curso de Farmácia, Universidade Católica de Brasília. Campus I, QS 07, Lote 01, EPCT, Águas Claras. Brasília, DF, Brasil. 71966-700. debora.farmacia9@ gmail.com

(b) Curso de Farmácia Universidade de Brasília. Asa Norte, DF, Brasil. daydelane@gmail.com

(c) Curso de Farmácia, Universidade Federal de Santa Catarina. Florianópolis, SC, Brasil. silvana.nair@ hotmail.com 


\section{Introdução}

O processo de envelhecimento populacional implica uma série de modificações nos aspectos físicos, psíquicos e sociais do ser humano, levando, na maioria das vezes, à necessidade de ser cuidado'.

No Brasil, o envelhecimento da população tem suscitado diversos debates, especialmente sobre o aumento das demandas dos serviços de saúde, bem como o desafio de buscar alternativas de cuidado para um crescente número de idosos².

De acordo com os pressupostos de humanização da assistência à saúde em gerontologia, emergiu a necessidade de ascensão das práticas de recuperação, reabilitação e promoção da saúde do idoso dentro do contexto domiciliar, ampliando o número de familiares engajados na prestação dos cuidados de saúde a esses pacientes.

O "Guia prático do cuidador", publicado em 2008 pelo Ministério da Saúde , estabelece a seguinte definição para o termo cuidador: "alguém que cuida a partir de objetivos estabelecidos por instituições especializadas ou responsáveis diretos, podendo ser da família ou da comunidade para prestar cuidados à outra pessoa de qualquer idade que esteja necessitando" (p. 8).

Embora existam diversas classificações dos tipos de cuidadores, tipifica-se, sobretudo, a categorização em "formal" e "informal". A Organização Mundial da Saúde (OMS) ${ }^{4}$ classifica o cuidador formal como aquele profissional que possui vínculo empregatício para a prestação do cuidado. Já o cuidador informal é aquele que desempenha o cuidado sem formalização trabalhista.

Dentre as funções desempenhadas pelos cuidadores de idosos, inclui-se a conduta do tratamento medicamentoso ${ }^{5}$. Esta atividade pode ser entendida como uma ampla assistência à terapia medicamentosa, compreendendo: a administração dos medicamentos, as ações e processos para garantia do acesso, a contemplação das recomendações técnicas para o armazenamento adequado, a observação da efetividade do tratamento, a comunicação e busca de esclarecimentos acerca das informações referentes à farmacoterapia com os profissionais de saúde, entre outras funções.

No cuidado da população idosa, a polifarmácia, a automedicação, o uso abusivo de outras formas de tratamento (como fitoterápicos, produtos homeopáticos, plantas medicinais, por exemplo) e a falta de adesão ao tratamento, compõem fatores presentes na farmacoterapia que acentuam a sua complexidade ${ }^{6,7}$. Como resultado, observa-se que a integração de todos esses fatores, adicionados às demais atividades do cuidado, contribui para que a atenção ao idoso, na prática da família cuidadora, se torne ainda mais desafiadora.

Mesmo com a ampliação dos investimentos financeiros no segmento da assistência farmacêutica - no Brasil, nos últimos anos ${ }^{8}$-, sobretudo como consequência dos direitos provenientes do desenvolvimento de políticas públicas nesse cenário, ainda persistem iniquidades no acesso aos medicamentos. Entender como esse e outros fatores presentes na farmacoterapia dos idosos interferem na sua condução pelos cuidadores é crucial para a estruturação de práticas educativas compatíveis com as necessidades de capacitação e fomento, além de abranger um importante indicador para a estruturação de programas e políticas públicas em prol da promoção do uso racional de medicamentos.

Com base na problematização pertinente ao cuidado diário do idoso e na complexidade do manejo da farmacoterapia, este estudo tem o intuito de discutir, a partir de uma reflexão teórica da literatura, as práticas do cuidador no que concerne à conduta do tratamento medicamentoso. Baseada na perspectiva de ampliar os dados para a discussão e no fato de que muitas tarefas são compartilhadas pelo grupo, a revisão abordará todos os estudos que envolvam cuidadores de idosos, independente das classificações formal/informal ou qualquer outro tipo de categorização.

\section{Metodologia}

Trata-se de uma revisão integrativa da literatura, cujo percurso metodológico foi operacionalizado pelas etapas: identificação e formulação do problema, coleta de dados, avaliação dos estudos, análise e interpretação dos dados coletados, e apresentação dos resultados ${ }^{9,10}$. 
A coleta de artigos, objeto de estudo, foi processada na aplicação dos descritores: "cuidador" e "idoso", com o operador booleano "and", na biblioteca eletrônica Scientific Electronic Library Online (Scielo), em janeiro de 2013. Foram obtidos e analisados 65 resumos de artigos.

Para a seleção final dos artigos, os autores fizeram a análise do resumo segundo os critérios de inclusão preestabelecidos: ter sido publicado entre 2003 e 2013, estar escrito em inglês e/ou português, e apresentar dados de interesse para a pesquisa, ou seja, relacionado ao objetivo do estudo. Após esse processo, foram adquiridas 23 referências. O percurso de recrutamento dos artigos está ilustrado na Figura 1.

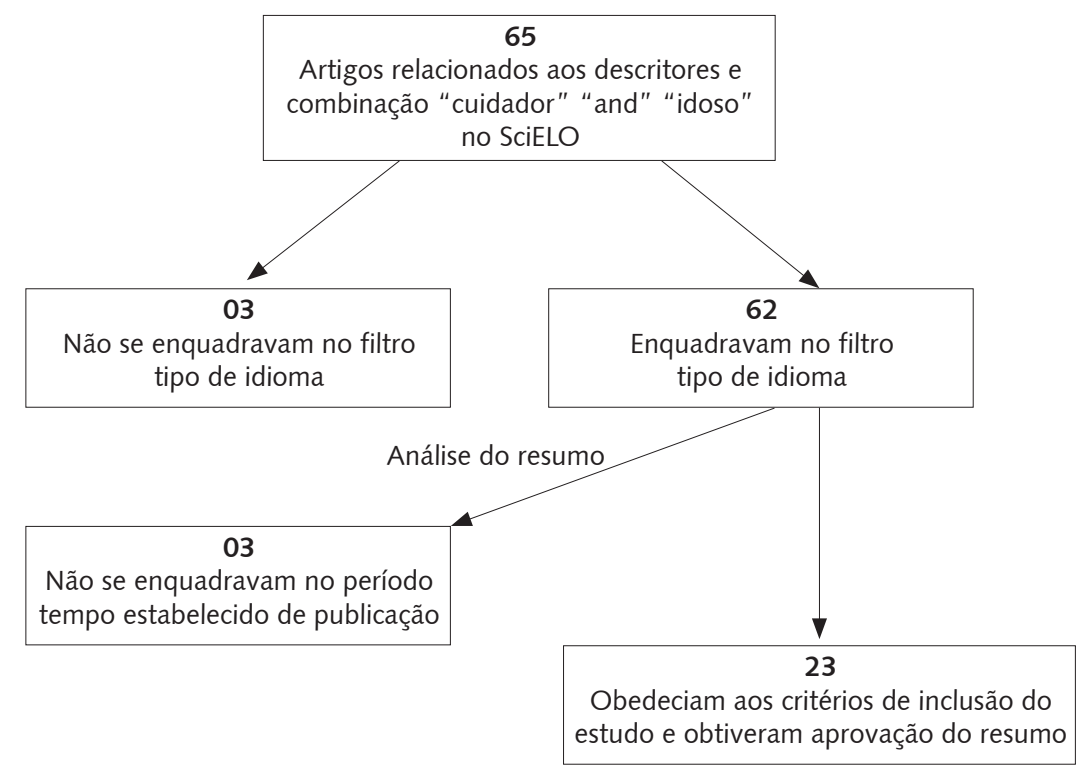

Figura 1. Etapas de recrutamento dos artigos

Conforme os critérios de busca e resultado da análise dos artigos, foram recrutados os seguintes estudos para dar sustentação à redação da revisão integrativa: Martins et al. ${ }^{5}$, Gaioli et al. ${ }^{11}{ }^{1}$, Klock et al. ${ }^{12}$, Carneiro e França ${ }^{13}$, Saliba et al. ${ }^{14}$, Rodrigues et al. ${ }^{15}$, Vieira et al. ${ }^{16}$, Moreira e Caldas ${ }^{17}$, Amendola et al. ${ }^{18}$, Gonçalves et al. ${ }^{19}$, Santos e Pavarini ${ }^{20}$, Figueiredo et al. ${ }^{21}$, Ribeiro et al. ${ }^{22}$, Silveira et al. ${ }^{23}$, Diogo et al. ${ }^{24}$, Paniz et al. ${ }^{25}$, Aziz et al. ${ }^{26}$, Veras et al. ${ }^{27}$, Fernandes e Garcia ${ }^{28}$, Nascimento et al. ${ }^{29}$, Santana et al. ${ }^{30}$, Marin et al. ${ }^{31}$, Monteschi et al. ${ }^{32}$.

Para a organização das discussões sobre a conduta do tratamento medicamentoso presente na literatura nacional, a partir do processo de análise textual qualitativa proposto no estudo de Oliveira ${ }^{33}$, foram identificados e isolados os seguintes enunciados de conteúdos: "caracterização dos cuidadores de idosos no Brasil", "acesso aos medicamentos", "administração de medicamentos" e "observação clínica e comunicação sobre a farmacoterapia". 


\section{O papel e perfil dos cuidadores de idosos no Brasil}

Em relação ao perfil dos cuidadores de idosos no Brasil, é possível observar que ainda segue hegemônica a concepção do cuidado como tarefa da mulher, assumindo, assim, o papel social de cuidar de alguém ${ }^{11-14}$.

Na contemporaneidade, a mulher está ganhando espaço social no mercado de trabalho. Sobretudo, é fundamental destacar o seu protagonismo quando se analisa o cuidado como campo de geração de emprego e renda ou, até mesmo, na atenção à saúde informal ao paciente ${ }^{12}$.

A média de idade dos cuidadores de idosos varia de acordo com o local de realização do estudo. Rodrigues et al. ${ }^{15}$ apontaram que grande parte dos cuidadores familiares também está na terceira idade, e que eles tinham de uma a quatro doenças crônicas, por vezes associadas a alguma incapacidade funcional. Dessa forma, essa situação ilustra que um idoso pode assumir as responsabilidades sobre o cuidado de outro idoso ${ }^{16}$. Moreira e Caldas ${ }^{17}$ destacam que, normalmente, o idoso independente é quem presta o cuidado ao idoso em condição de dependência.

A literatura ilustra que a escolaridade dos cuidadores é predominantemente baixa ${ }^{16,18,19}$. Santos e Pavarini ${ }^{20}$ destacam que o baixo nível de escolaridade interfere, direta ou indiretamente, na prestação de cuidados aos idosos, configurando uma barreira no processo de educação em saúde.

Figueiredo et al. ${ }^{21}$, em seu estudo com cuidadores familiares de idosos, encontraram que $78,8 \%$ da amostra possuem o estado civil casado ou em união estável. Ribeiro et al. ${ }^{22}$ destacam que a variabilidade de estado civil na amostra de cuidadores acompanha a variável idade, sendo que, em locais de realização de pesquisa cuja amostra tem idade mais avançada, observa-se que o padrão do estado civil tende a ser casado ou viúvo.

As atividades exercidas por cuidadores tendem a ser assumidas por uma única pessoa, denominada de cuidador principal ${ }^{23}$. Essa pessoa assume e se responsabiliza pelas tarefas de cuidado, incluindo as atividades de: higiene, alimentação, supervisão domiciliar, acompanhamento nos serviços de saúde, condução da terapia medicamentosa, apoio na prática de exercícios físicos, entre outras, que surgirem em função da dependência e necessidades de saúde do idoso. Com frequência, esses cuidadores se sobrecarregam, pois não possuem ajuda de outro membro da família ou dos serviços de saúde. Logo, centraliza-se, na pessoa do cuidador, o elo entre idoso, família e a equipe de saúde ${ }^{24}$.

Os cuidadores familiares constituem uma rede autônoma de atendimento ao idoso, sem a devida integração com os serviços de saúde. Esses atores, frequentemente, conduzem os idosos aos atendimentos de saúde sem compreender a organização e articulação dos diversos níveis de assistência à saúde, carecendo, também, de informações sobre quais profissionais e serviços devem ser solicitados na busca de apoio e orientações ${ }^{17}$. A compreensão de como o sistema de saúde se organiza, na lógica de redes de atenção à saúde, colabora para que o cuidado seja qualificado e que o paciente e cuidador tenham as suas demandas e necessidades atendidas no contexto da integralidade de acordo com os serviços e momentos oportunos. Dessa forma, o empoderamento do cuidador deve compreender todos esses eixos, otimizando a dinâmica do cuidado, além de facilitar o fluxo desses usuários no Sistema Único de Saúde (SUS).

\section{Acesso aos medicamentos: um entrave persistente na assistência à saúde do idoso no domicílio}

O acesso a medicamentos é um indicador da qualidade e resolutividade do sistema de saúde ${ }^{25}$. No contexto da farmacoterapia do idoso, caracterizada pela alta necessidade de consumo de medicamentos, se comparado a outros estratos populacionais, a garantia do acesso torna-se ainda mais desafiadora para as famílias e sistema de saúde ${ }^{26}$.

Veras et al. ${ }^{27}$, em um estudo transversal de avaliação de gastos financeiros pelas famílias com idosos com demência, demonstraram que o cuidado domiciliar tem grande repercussão financeira. Dentro da estrutura de gastos, o item que constitui maior custo é a compra de medicamentos para o idoso. 
As famílias de idosos com prejuízo cognitivo, alterações do comportamento e diagnóstico de síndrome demencial podem apresentar maior comprometimento do orçamento familiar conforme se processa a evolução da severidade da doença ou, até mesmo, com o surgimento de comorbidades ${ }^{27}$.

Fernandes e Garcia ${ }^{28}$ detectaram, no relato dos cuidadores, que, nos recursos materiais de consumo pelo paciente idoso, prevaleciam: o uso de fraldas $(73,7 \%)$, seguido pela necessidade de uso de medicamentos de maneira sistemática e contínua (50\%). Esse mesmo estudo traz que, por carência econômica, 40\% dos pacientes faziam interrupções na utilização da terapia, e 3,3\% nunca podiam comprar tais substâncias, apesar de necessitar. Esses dados mostram claramente o impacto que a falta de acesso gera no aumento da fragilidade do idoso e o comprometimento da adesão à medicação.

Gonçalves et al. ${ }^{19}$, Moreira e Caldas ${ }^{18}$ ressaltam que a sobrecarga do cuidador possui etiologia multifatorial, e tem sido associada aos problemas físicos, psicológicos, sociais e financeiros que podem ser vivenciados no cuidado diário do idoso. Assim, a carência financeira da família e a falta de suporte estatal, além de prejudicarem o acesso aos medicamentos e a qualidade da assistência à saúde do idoso, podem acarretar comprometimento da saúde física e mental dos cuidadores.

Os medicamentos são tecnologias em saúde indispensáveis para as práticas de recuperação e reabilitação da saúde do idoso em cuidado domiciliar. A utilização de programas na área de assistência farmacêutica, pela família e paciente, é fundamental para o fornecimento oportuno desses produtos. Também é importante destacar a atuação do programa farmácia popular que, de maneira paralela e complementar, contribui na oferta de medicamentos comumente utilizados pelo público idoso, sobretudo aqueles que são indicados para o tratamento das doenças crônicas não transmissíveis. Contudo, o baixo financiamento do setor de saúde no Brasil, que tem impacto direto e acarreta o orçamento estreito previsto para a compra de medicamentos e outros produtos para saúde, acaba sobrecarregando, ainda mais, o cuidado domiciliar. Esse quadro culmina na reorganização do planejamento financeiro da família, sendo a verba destinada, em grande parcela, para a compra desses produtos, em detrimento da aquisição de outros bens e serviços elementares para a promoção da saúde e qualidade de vida, como o acesso a alimentação saudável e às práticas de lazer, por exemplo.

\section{Administração de medicamentos: os desafios da utilização}

Nascimento et al. ${ }^{29}$, Martins et al. ${ }^{5}$ e Vieira et al. ${ }^{16}$ abordam que as práticas inerentes à medicação fazem parte do rol de atividades frequentemente prestadas pelos cuidadores de idosos. Não obstante, ainda são escassos os estudos que se dedicam à análise e ao impacto da responsabilidade diária da administração de medicamentos no cuidado de um idoso dependente.

Os idosos que apresentam alto grau de dependência precisam de cuidadores que se responsabilizem pela administração de diversas doses durante o dia ${ }^{31}$. Consolidar o esquema de administração com as rotinas inerentes ao cuidado e ao cotidiano da família, tornou-se viável para os cuidadores que aboliram outras responsabilidades para se dedicarem exclusivamente à tarefa do cuidado. Quando os cuidadores trabalham ou possuem outra responsabilidade, é detectada maior quantidade de horários perdidos de administração, e, portanto, menor adesão ao tratamento ${ }^{30}$.

Para os cuidadores que se mantêm ativos no mercado de trabalho, é imprescindível receber ajuda de parentes e empregados domésticos no cuidado do idoso, havendo rodízios do desempenho e supervisão da administração de medicamentos ${ }^{28}$.

Fernandes e Garcia ${ }^{28}$ discutem que o diminuto ou, até mesmo, inexistente acesso aos medicamentos resulta o agravamento progressivo da saúde e a geração de incapacidade dos utentes. Como consequência, ampliam-se as necessidades desses idosos em termos de demanda de cuidados, acarretando o surgimento ou agravamento de enfermidades. Esse processo ainda possui como desfecho, a intensificação da necessidade e quantidade de medicamentos a serem consumidos pelo idoso, o que gera dificuldades adicionais para os cuidadores implementarem suas ações. A prática da administração de medicamentos constitui um exemplo de atividade instrumental de vida diária que, no cuidado do idoso dependente e/ou com incapacidades, é amplamente executada pelos 
cuidadores ${ }^{13}$. Sobretudo o paciente polimedicado, com uso de dispositivos complexos para a utilização de medicamentos, que necessita de produtos administrados por vias complexas (como no caso das parenterais), é fundamental o apoio prestado pelos profissionais de saúde por meio das atividades de educação em saúde, de forma a qualificar a assistência à saúde do utente oferecida pelos respectivos cuidadores

\section{Observação clínica e comunicação sobre a farmacoterapia}

É de competência dos cuidadores o acompanhamento dos seus idosos aos serviços de saúde. Nesse momento, o familiar atua como importante emissor de informações sobre a saúde do idoso e, ao se comunicar com os profissionais de saúde, ele colabora na realização de uma avaliação clínica efetiva ${ }^{16}$. Por consequência, quando os serviços de saúde pretendem investigar a adequação da medicação do idoso, cabe sobretudo ao cuidador relatar as alterações comportamentais e funcionais do paciente, de modo a subvencionar os julgamentos clínicos da farmacoterapia pelos profissionais de saúde.

Carneiro e França ${ }^{13}$ investigaram os conflitos no relacionamento entre os cuidadores e seus idosos. Foi encontrado que os idosos que não possuíam companheiros(as) apresentaram maior nível de conflito com seus cuidadores do que idosos que viviam com companheiros(as). Foi proposto pelos autores que fossem desenvolvidas estratégias para a redução destes conflitos, como a estimulação da oferta de cursos para a melhoria das habilidades de comunicação interpessoal entre cuidadores e idosos, por exemplo.

Santana et a ${ }^{30}$ destacam que "o sujeito que sofre de síndrome demencial apresenta problemas na comunicação como retardamento no tempo de resposta, problemas de memória, déficits sensoriais e flutuações do pensamento". Todos esses fatores associados com o quadro patológico da demência, além das limitações decorrentes do processo de envelhecimento natural, prejudicam, ainda mais, a comunicação entre familiares e idosos.

Segundo Marin et al. ${ }^{31}$, as reações adversas a medicamentos são 2,5 vezes mais frequentes nos idosos do que em outras faixas etárias. Quando há prejuízo na capacidade de comunicação entre cuidador e idoso, as captações dessas informações ficam subjugadas.

Diante desse cenário, os profissionais de saúde devem capacitar os cuidadores de idosos para a observação das alterações fisiológicas/patológicas do idoso em uso de medicamentos, bem como estabelecer diálogo horizontal e democrático, criando espaços para que os cuidadores possam falar e ser atentamente ouvidos, por meio da escuta qualificada. Essa prontidão na busca ativa e coleta sistemática de informações, a partir das narrativas dos cuidadores, auxiliará os profissionais de saúde na detecção e classificação das reações adversas a medicamentos, constituindo ferramentas indispensáveis nas ações de farmacovigilância.

Monteschi et al. ${ }^{32}$ detectaram, nos depoimentos dos cuidadores de idosos com transtorno afetivo bipolar, preocupação com efeitos adversos e inefetividade terapêutica. Os aspectos relacionados à falta de efetividade e segurança são mencionados como agentes causais de intervenções do esquema terapêutico pelos cuidadores.

Os profissionais de saúde tendem a condenar as intervenções dos cuidadores sobre a farmacoterapia, e, por essa razão, muitas vezes, elas não são relatadas nos atendimentos. No entanto, não se pode condenar um cuidador que age dessa forma na busca do bem-estar do idoso. Os profissionais de saúde, ao entrarem na realidade do cuidador, deixando de lado posturas taxativas e discriminatórias, poderão extrair essas informações para a realização de intervenções efetivas e contextualizadas, incluindo a estruturação e oferta de ações educativas em favor do empoderamento desses cuidadores ${ }^{16}$.

A comunicação entre o cuidador e o idoso é muito importante para a avaliação clínica, e a transmissão dessas informações é essencial para os profissionais de saúde. No entanto, essa troca de informações deve inserir as concepções dos idosos, sendo necessária a pactuação das preferências de cuidado que eles desejam receber do seu familiar e dos serviços de saúde. 


\section{Considerações finais}

De acordo com a análise das produções científicas, foi possível identificar e discutir alguns dos principais fatores dentro do contexto da conduta do tratamento medicamentoso por cuidadores de idosos. Esses dados são importantes para fornecer subsídios em prol de uma assistência à saúde multidimensional, integral e qualificada para o idoso e para a família. Contudo, a produção científica e técnica pouco se atenta ao medicamento como um importante instrumento no cuidado, sendo a escassez de estudos nessa área uma limitação importante para a elaboração de estudos de revisão.

Sobre a administração de medicamentos pelos cuidadores de idosos, os estudos revelam que, dentre as principais dificuldades para o uso seguro dos medicamentos, está a necessidade de negociação entre a rotina da família e os horários de utilização dos medicamentos.

Para assegurar o acesso ao tratamento medicamentoso aos idosos sob cuidado, é essencial a estruturação e implantação de políticas de saúde que certifiquem o suprimento dessas necessidades. Embora muitos medicamentos sejam ofertados para o tratamento de várias doenças nos idosos por meio dos componentes básico, estratégico e especializado da assistência farmacêutica, assim como pelo programa farmácia popular, a descontinuidade do abastecimento e a prescrição de medicamentos não padronizados culminam na transferência da responsabilidade da compra dos medicamentos para as famílias, resultando em: comprometimento da renda familiar; descumprimento dos esquemas terapêuticos, terapêutica irracional, falta de adesão ao tratamento, comprometimento da saúde dos idosos, e piora da sobrecarga e saúde mental dos cuidadores.

Para a avaliação da efetividade e segurança do tratamento proposto, é importante a compilação de dados que se refiram às alterações clínicas do paciente. Os cuidadores devem ser empoderados a efetuar observações clínicas do idoso e relatá-las com liberdade para a equipe de saúde, articulando uma parceria entre a família e esses profissionais. É importante o engajamento dos diversos cuidadores nessa tarefa, para diminuir o viés da aquisição de informações sob um único prisma que poderia tender a decisões clínicas inapropriadas e incompletas, o que fragilizaria o princípio de atendimento integral da pessoa idosa.

No Brasil, a maior parte dos estudos que abordam os fatores que interferem no uso racional de medicamentos pela população de terceira idade utiliza como participante os próprios idosos, isoladamente, como se eles fossem os únicos participantes da conduta do tratamento medicamentoso. No entanto, na mesma proporção de surgimento e consolidação do envelhecimento populacional, amplia-se o engajamento das famílias na execução de tarefas voltadas para a medicação, e elas, por sua vez, estão sendo negligenciadas como agentes importantes nesse processo.

Diante desse quadro, é imprescindível a realização de estudos que revelem os obstáculos a serem superados por essas famílias no que se refere à conduta do tratamento medicamentoso, para que, a partir desses pressupostos, sejam elencadas as intervenções de educação em saúde e políticas públicas que ofereçam apoio à família cuidadora de idosos.

\section{Colaboradores}

Débora Santos Lula Barros participou na elaboração do artigo, enquanto Dayde Lane Mendonça e Silvana Nair Leite trabalharam na revisão do texto. 


\section{Referências}

1. Novelli MMPC, Nitrin R, Caramelli P. Cuidadores de idosos com demência: perfil sociodemográfico e impacto diário. Rev Ter Ocup Univ São Paulo. 2010; 21(2):139-47.

2. Duca GF, Thumé E, Hallal PC. Prevalência e fatores associados ao cuidado domiciliar a idosos. Rev Saude Publica. 2011; 45(1):113-20.

3. Ministério da Saúde. Secretaria de Atenção à Saúde. Secretaria de Gestão do Trabalho e da Educação na Saúde. Guia prático do cuidador. Brasília (DF): MS; 2008.

4. Organização Mundial da Saúde. Envelhecimento ativo: uma política de saúde [Internet]. 2005 [acesso 2012 Nov 18]. Disponível em: http://portal.saude.gob.br/portal/arquivos/ pdf/envelhecimento_ativo.pdf

5. Martins JJ, Albuquerque GL, Nascimento ERP, Barra DCC, Souza WGA, Pacheco WNS. Necessidades de educação em saúde dos cuidadores de pessoas idosas no domicílio. Texto Contexto - Enferm. 2007; 16(2):254-62.

6. Correr CJ, Pontarolo R, Ferreira LC, Baptistão SAM. Riscos de problemas relacionados com medicamentos em pacientes de uma instituição geriátrica. Rev Bras Cienc Farm. 2007; 43(1):55-62.

7. Secoli SR. Polifarmácia: interações e reações adversas no uso de medicamentos por idosos. Rev Bras Enferm. 2010; 63(1):136-40.

8. Vieira FS, Zucchi P. Financing of pharmaceutical services in Brazilian public health system. Saude Soc. 2013; 22(1):73-84.

9. Mendes KDS, Silveira RCCP, Galvão CM. Revisão integrativa: método de pesquisa para a incorporação de evidências na saúde e na enfermagem. Texto Contexto - Enferm. 2008; 17(4):758-64.

10. Sousa LD, Filho-Lunardi WD, Lunardi VL, Santos SSC, Santos CP. A produção científica de enfermagem acerca da clínica: uma revisão integrativa. Rev Esc Enferm USP. 2011; 45(2):494-500.

11. Gaioli CCLO, Furegato ARF, Santos JLF. Perfil de cuidadores de idosos com doença de alzheimer associado à resiliência. Texto Contexto - Enferm. 2012; 21(1):150-7.

12. Klock AD, Heck RM, Casarim ST. Cuidado domiciliar: a experiência da residência multiprofissional em saúde da família/UFPEL-MS/BID. Texto Contexto - Enferm. 2005; 14(2):237-45.

13. Carneiro VL, França LHFP. Conflitos no relacionamento entre cuidadores e idosos: o olhar do cuidador. Rev Bras Geriatr Gerontol. 2011; 14(4):647-62.

14. Saliba NA, Moimaz SAS, Marques JAM, Prado RL. The profile of caregivers for the elderly and oral health perception. Interface (Botucatu). 2007; 11(21):39-50.

15. Rodrigues SLA, Watanabe HAW, Derntl AM. A saúde de idosos que cuidam de idosos. Rev Esc Enferm USP. 2006; 40(4):493-500.

16. Vieira CPB, Fialho AVM, Freitas CHA, Jorge MSB. Práticas do cuidador informal do idoso no domicílio. Rev Bras Enferm. 2011; 64(3):570-9.

17. Moreira MD, Caldas CP. A importância do cuidador no contexto da saúde do idoso [Internet]. Esc Anna Nery [acesso 2013 Jan 10]. 2007; 11(3):520-5. Disponível em: http://www.scielo.br/scielo.php?pid=S1414-81452007000300019\&script=sci_arttext

18. Amendola F, Oliveira MAC, Alvarenga MRM. Qualidade de vida dos cuidadores de pacientes dependentes no programa de saúde da família. Texto Contexto - Enferm. 2008; 17(2):266-72.

19. Gonçalves LHT, Alvarez AM, Sena ELS, Santana LWS, Vicente FR. Perfil da família cuidadora de idoso doente/fragilizado do contexto sociocultural de Florianópolis, SC. Texto 
Contexto - Enferm. 2006; 15(4):570-7.

20. Santos AA, Pavarini SCl. Perfil dos cuidadores de idosos com alterações cognitivas em diferentes contextos de vulnerabilidade social. Rev Gaucha Enferm. 2010; 31(1):115-22.

21. Figueiredo D, Lima MP, Sousa L. Cuidadores familiares de idosos dependentes com e sem demência: rede social, pessoal e satisfação com a vida. Psic Saude Doenças. 2012; 13(1):117-29.

22. Ribeiro MTF, Ferreira RC, Ferreira E, Magalhães CS, Moreira AN. Perfil dos cuidadores de idosos nas instituições de longa permanência de Belo Horizonte, MG. Cienc Saude Colet. 2008; 13(4):1285-92.

23. Silveira TM, Caldas CP, Carneiro TF. Cuidando de idosos altamente dependentes na comunidade: um estudo sobre cuidadores familiares principais. Cad Saude Publica. 2006; 22(8):1629-38.

24. Diogo EJD, Ceolim MF, Cintra FA. Orientações para idosas que cuidam de idosos no domicílio: relato de experiência. Rev Esc Enferm USP. 2005; 39(1):97-102.

25. Paniz VMV, Fassa AG, Facchini LA, Bertoldi AD, Piccini RX, Tomasi E, et al. Acesso a medicamentos de uso contínuo em adultos e idosos nas regiões Sul e Nordeste do Brasil. Cad Saude Publica. 2008; 24(2):267-80.

26. Aziz MM, Calvo MC, Schneider IJC, Xavier AJ, d'Orsi E. Prevalência e fatores associados ao acesso a medicamentos pela população idosa em uma capital do sul do Brasil: um estudo de base populacional. Cad Saude Publica. 2011; 27(10):1939-50.

27. Veras RP, Caldas CP, Dantas SB, Sancho LG, Sicsú B, Motta LB, et al. Avaliação dos gastos com o cuidado do idoso com demência. Rev Psiquiatr Clin. 2007; 34(1):5-12.

28. Fernandes MGM, Garcia TR. Atributos da tensão do cuidador familiar de idosos dependentes. Rev Esc Enferm USP. 2009; 43(4):818-24.

29. Nascimento LC, Moraes ER, Silva JC, Veloso LC, Vale ARMC. Cuidador de idosos: conhecimento disponível na base de dados LILACS. Rev Bras Enferm. 2008; 61(4):514-7.

30. Santana RF, Figueiredo NMA, Ferreira MA, Alvim NAT. A formação da mensagem na comunicação entre cuidadores e idosos com demência. Texto Contexto - Enferm. 2008; 17(2):288-96

31. Marin MJS, Cecílio LCO, Perez AEWUF, Santella F, Silva CBA, Filho-Gonçalves JR, et al. Caracterização do uso de medicamentos entre idosos de uma unidade do Programa Saúde da Família. Cad Saude Publica. 2008; 24(7):1545-55.

32. Monteschi M, Vedana KGG, Miasso Al. Terapêutica medicamentosa: conhecimento e dificuldades de familiares de pessoas idosas com transtorno afetivo bipolar. Texto Contexto - Enferm. 2010; 19(4):709-18.

33. Oliveira DC. Análise de conteúdo temático-categorial: uma proposta de sistematização. Rev Enferm UERJ. 2008; 16(4):569-76. 
Barros DSL, Mendonça-Silva DL, Leite SN. Conducta del tratamiento medicamentoso por cuidadores de ancianos. Interface (Botucatu). 2015; 19(54):527-36.

El envejecimiento poblacional está ampliando el número de cuidadores de ancianos en Brasil. La farmacoterapia incluye un sistema complejo de actividades y su realización está presente en las diversas tareas desempeñadas por esos actores. Además, la conducta del tratamiento puede estar sujeta a diversos problemas en su realización, destacándose los siguientes: las dificultades de administración de los medicamentos, la falta de acceso a los medicamentos, los obstáculos en la realización de observación clínica, así como los desafíos presentes en el establecimiento de una comunicación efectiva con los ancianos y profesionales de la salud. El presente estudiose trata de una revisión cuyo objetivo es ampliar las discusiones sobre los aspectos anteriormente citados, de forma que tal debate proporcione subsidios teóricos a los programas y servicios en el área de la gerontología.

Palabras clave: Medicamentos. Anciano. Cuidadores. Conducta del tratamiento medicamentoso.

Recebido em 23/02/14. Aprovado em 30/10/14. 